\title{
NESTED VARIANT OF UROTHELIAL CARCINOMA OF RENAL PELVIS - A CASE REPORT
}

\author{
Piotr Kuzaka ${ }^{1}$, Krzysztof Pastewka ${ }^{1}$, Jan Faryna ${ }^{2}$, Boleseaw Kuzaka ${ }^{3}$, Wojciech Pypno ${ }^{1}$, \\ JAROSEAW WEJMAN ${ }^{2}$
}

\author{
${ }^{1}$ Department of Urology, Professor Witold Orlowski Clinical Hospital, Center for Medical Postgraduate Education, \\ Warsaw, Poland \\ ${ }^{2}$ Department of Pathology Postgraduate, Professor Witold Orlowski Clinical Hospital, Center for Medical Postgraduate \\ Education, Warsaw, Poland
}

\begin{abstract}
We report a case of an 80-year-old woman with a very rare subtype of urothelial carcinoma - nested variant of urothelial carcinoma mimicking physiological von Brunn's nests. Optimal treatment of NVUC has not been determined due to the small number of cases, as well as the lack of randomized and follow-up studies. In our case the right retroperitoneal nephroureterectomy was chosen.
\end{abstract}

Key words: urothelial carcinoma, NVUC, renal pelvis.

\section{Introduction}

Urothelial tumors of the upper urinary tract are rare. The majority of them are transitional epithelial tumors, located in the urinary bladder (93\%), the ureterocalyceal system $(4 \%)$, and in ureters (3\%) [1]. Neoplasms presenting histological features of nested variant of urothelial carcinoma (NVUC) may have similar location. Diagnosis of NVUC is based on the histological picture of the tumor, which is characterized by the presence of benign-looking urothelial carcinoma cells occupying the lamina propria and/or infiltrating the muscular layer. In spite of its bland morphology, NVUC can have a very poor prognosis due to its aggressive behavior; therefore it is very important to distinguish this neoplasm from benign urothelial lesions such as von Brunn's nests, inverted papilloma and cystitis cystica.

\section{Case report}

An 80-year-old woman was admitted to the hospital due to recurrent lumbar right pain and associated hydronephrosis confirmed by ultrasonographic study. $\mathrm{X}$-ray study of the abdomen revealed no radioopaque concrements. Urinalysis showed mild hematuria and leukocyturia. Ureteropyelography showed dilatation of the ureterocalyceal system and the presence of an irregular mass in the right renal pelvis at the uretero-pelvic junction (Fig. 1)

CT scan confirmed the presence of tumor in the renal pelvis, revealing a defect of the contrast filling, about $20 \times 9 \mathrm{~mm}$ in size, with delayed excretion of contrast medium (Fig. 2).

The patient underwent right retroperitoneal nephroureterectomy through Israel-Bergmann incision. Intraoperatively we found a hard and solid pelvic lesion with accompanying enlargement of the hilar lymph nodules. The postoperative course was uncomplicated and the patient was dismissed on the $10^{\text {th }}$ day after surgery, following her good general condition. About one and a half years later the woman died due to cardiovascular and respiratory insufficiency.

The specimen sent for histological examination was a kidney $12 \times 5.5 \times 5 \mathrm{~cm}$ in size. On cross section, an exophytic tumor, $3.5 \times 2 \times 2 \mathrm{~cm}$ in size, was found. It appeared to originate from the renal pelvis and was located at the pyeloureteric junction (Fig. 4). The cut surface of the tumor was solid and white-grayish, with areas of infiltration through the renal pelvis wall. The uretero-pyelo-caliceal system was dilated (Fig. 5). Ad- 


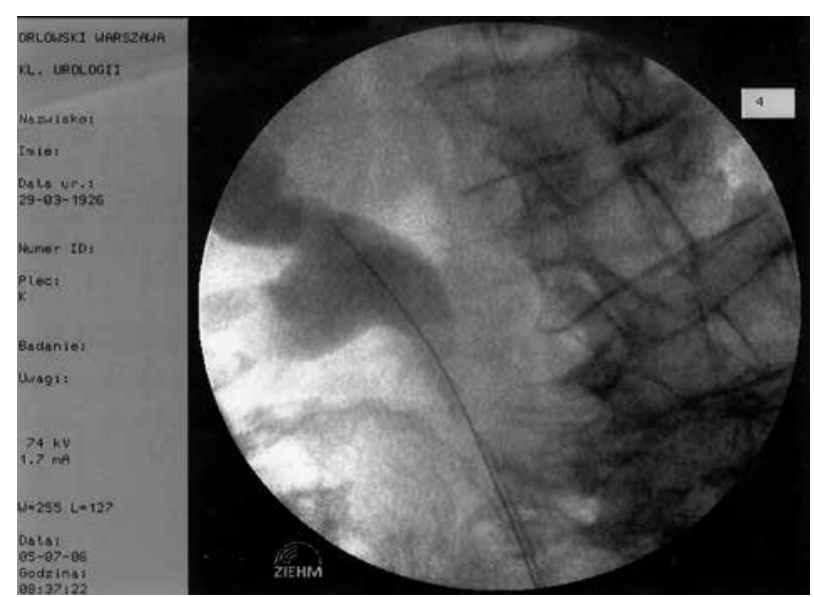

Fig. 1. Pyelography right. Dilated ureterocalyceal system right. Filling defect of contrast medium excretion at the uretero-pelvic junction. Presence of irregular mass in the renal right pelvis at the uretero-pelvic junction
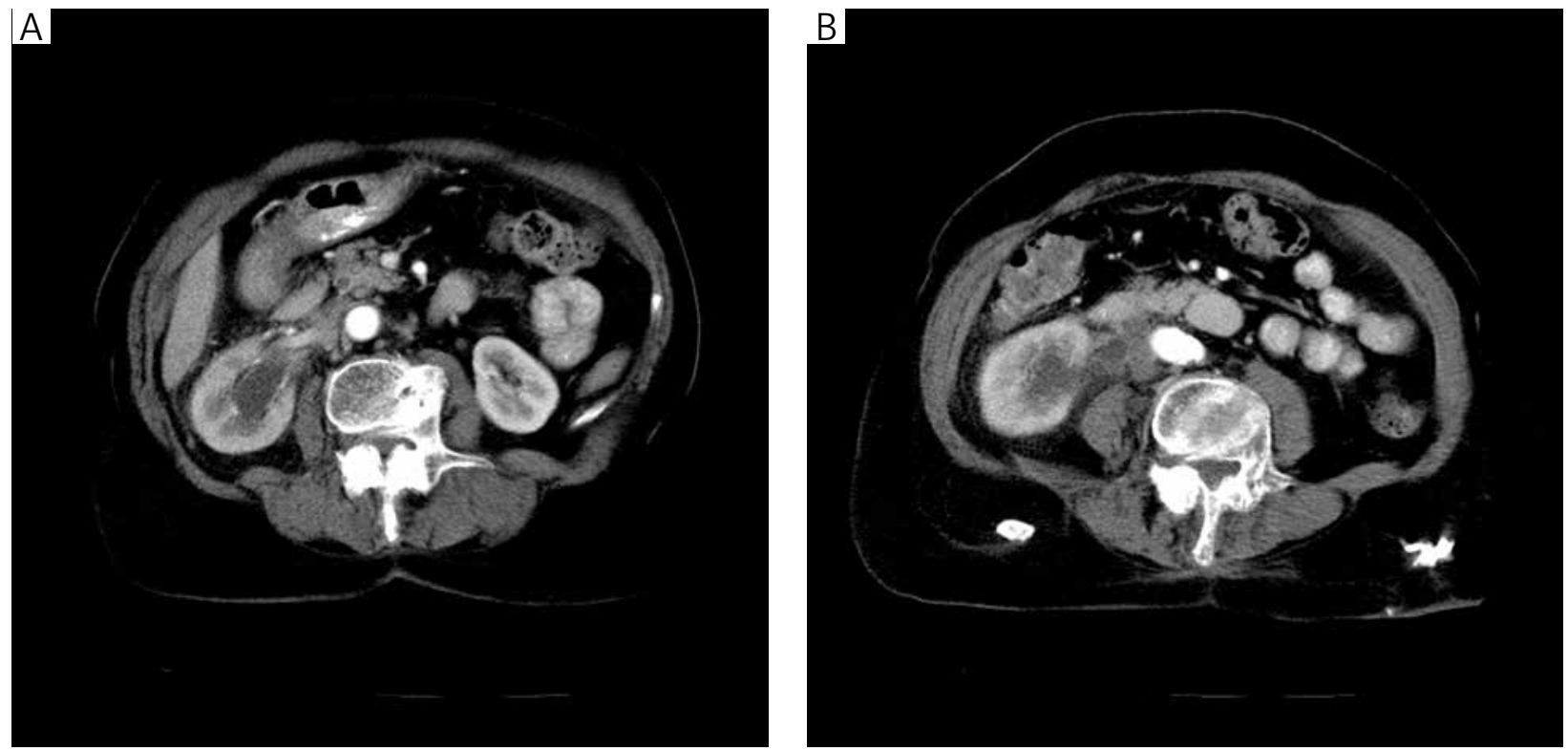

Fig. 2. CT scan - arterial phase - confirmed the diagnosis of right renal pelvis tumor

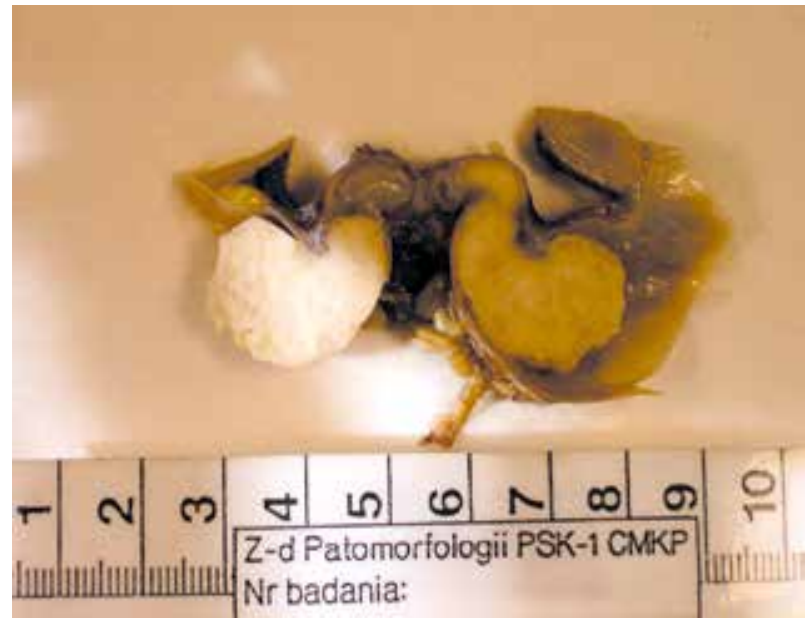

Fig. 3. Gross appearance of the "nested type" carcinoma of the renal pelvis - cross section of the kidney through the ureteropelvic junction ditionally, we found an oval, encapsulated fragment of tissue, $2.5 \mathrm{~cm}$ in diameter. On cross section the nodule was formed of solid white tissue, resembling a pathologically enlarged lymph node.

Microscopically the tumor was composed of nests of cells with signs of slight and mediocre atypia along with foci of scattered highly atypical urothelial cells. The tumor was located below the surface of normal urothelium and was infiltrating the full thickness of the pelvic wall. There were signs of ureteral and hilar fat involvement. Numerous lymph vessels were filled with neoplastic cells.

The surgical margin in the distal ureter was uninvolved.

A group of hilar lymph nodes with metastatic foci was also found.

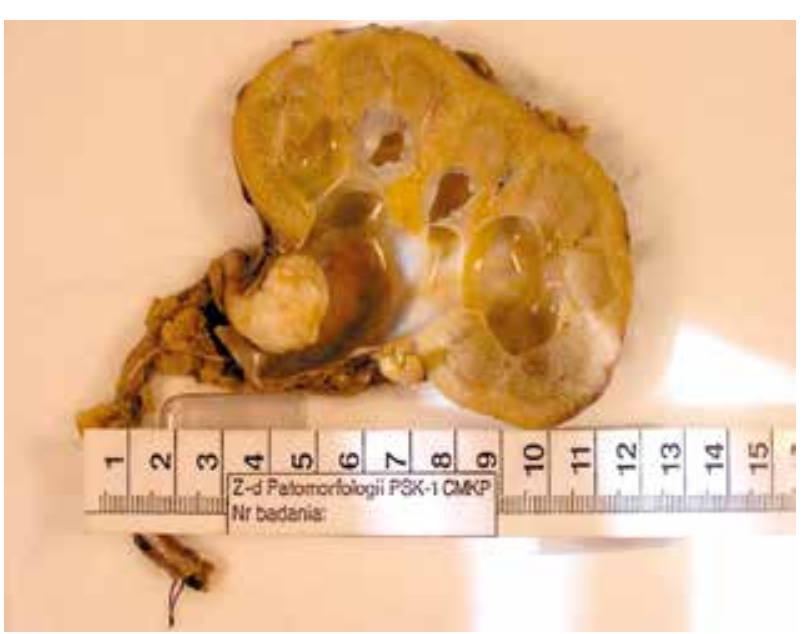

Fig. 4. Gross appearance of the "nested type" carcinoma of the renal pelvis 

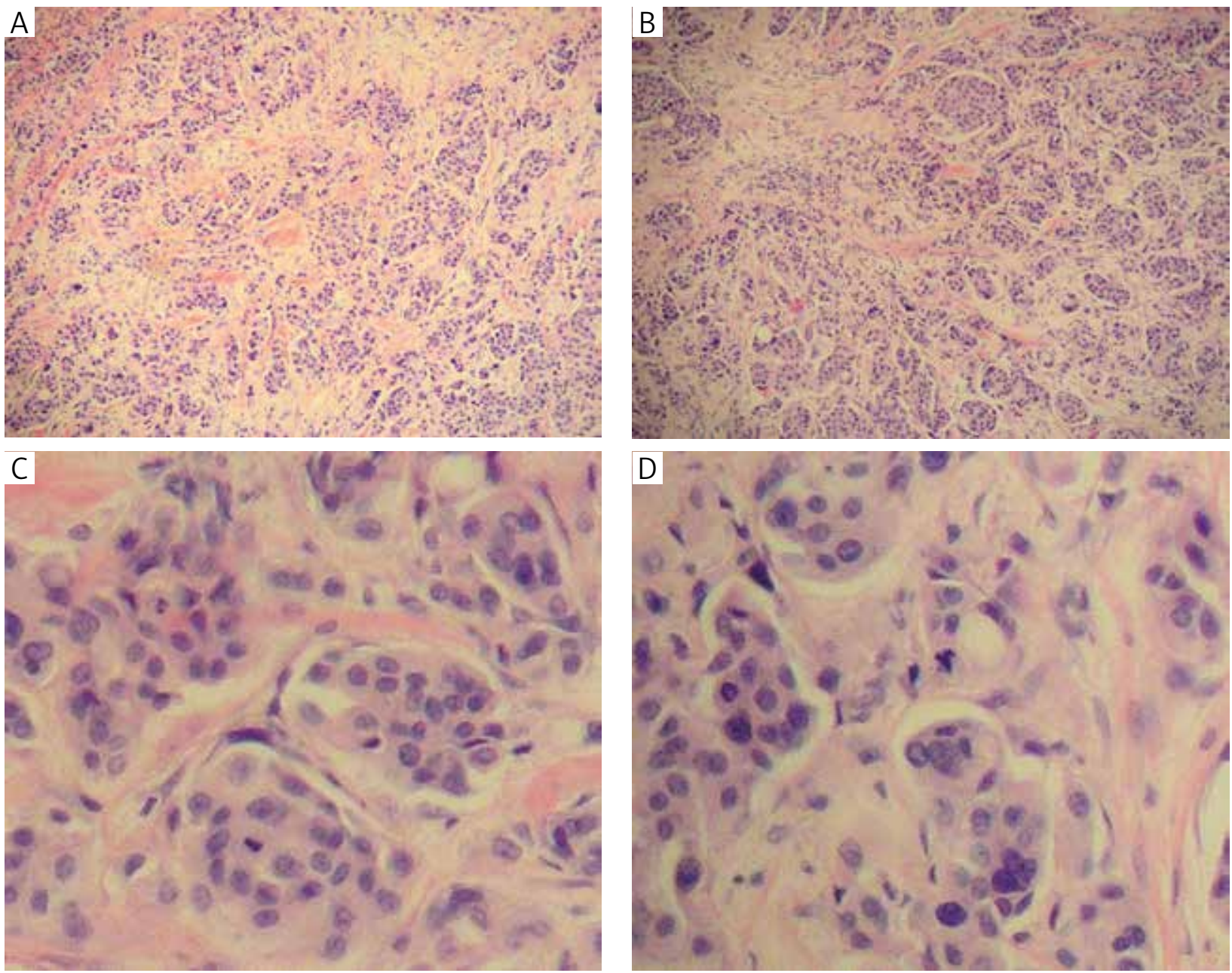

Fig. 5. Microscopic images of the NVUC with characteristic neoplastic cell aggregations - "von Brunn-like nests"

\section{Discussion}

According to the WHO definition, nested variant of urothelial carcinoma (NVUC) is a rare urothelial cancer with a great malignant potential. NVUC belongs to a group of rare urothelial tumors with unfavorable prognosis. This histologic group encompasses micropapillary, microcystic, glandular, sarcomatoid and squamous variants of urothelial carcinoma along with paraganglioma.

The first description of histologic features of NVUC was presented in 1979 by Stern, who considered it to be a benign tumor [2]. Talbert and Young [3], ten years later (1989), redefined the tumor as a malignant one. According to the literature, based on analysis of 75 cases (described in 15 publications), NVUC has a predilection for the urinary bladder and in rare cases the ureter. Pusztaszari et al. reported coexistence of NVUC in the renal pelvis and in the ureter [4], and Lau [5] and Tripodi et al. [6] described the tumor located only in the renal pelvis.

This type of neoplasm accounts for only about $0.8 \%$ of all urothelial cancers. Mortality is about $70 \%$. Patients survive from 4 to 40 months after the initial di- agnosis $[1]$ (mean 17.6 months). The reviewed literature suggests that NVUC has a clinical course similar to that of high-grade urothelial carcinoma. In the majority of cases the tumor is exophytic, rarely intramural, with infiltration of the normal urothelium. In our case there was partial infiltration of the renal pelvis wall. Pusztaszari et al. reported coexistence of NVUC in the renal pelvis and in the ureter [4], and Lau [5] and Tripodi et al. [6] reported it only in the renal pelvis.

The microscopic image of NVUC might be misdiagnosed as a benign group of von Brunn's nests. In our case the signs of tumor invasiveness and metastases into the regional lymph nodes confirmed the diagnosis of a malignant neoplasm.

The histological picture of the nested variant of urothelial carcinoma occasionally may also imitate paraganglioma. Usually paraganglioma is easily discernible due to the presence of a characteristic vascular network, and unlike NVUC exhibits positive immunoreactivity for neuroendocrine markers, such as chromogranin and synaptophysin, with no reactivity with anti-cytokeratin antibodies [5].

NVUC must also be differentiated from cystitis cystica and cystitis glandularis. Cystitis cystica et 
glandularis (CCG) arises from von Brunn nests in the lamina propria by developing slitlike and cystic spaces which have luminal and cuboidal or columnar lining. In the case of CCG glandular cells are seen, along with intestinal metaplasia and scattered intestinal-type goblet cells. Paneth cells and argentaffin cells may also be present. There is no destructive invasion of the muscularis propria. Cystitis cystica has invaginated nests in the superficial lamina propria, with cystic dilatation forming luminal spaces, and no cuboidal or columnar lining cells.

Nephrogenic metaplasia and inverted papilloma should also be considered in the differential diagnosis. Nephrogenic metaplasia has typically basal nuclear palisading around nests and may have cystitis cystica-like appearance.

Carcinoid, which should also be excluded, is usually formed by endophytic thin anastomosing cords of atypical cells, with more complex cribriform or cordlike growth pattern, and shows immunoreactivity for synaptophysin, serotonin and chromogranin.

Finally, some NVUC may resemble usual transitional cell carcinoma (TCC) of the bladder. Also urothelial carcinoma may show a nested pattern of invasion. In our case, no surface involvement (which is usually present in TCC) was seen.

Involvement of the renal parenchyma by the NVUC may sometimes lead to misdiagnosis with renal cell carcinoma (RCC). Neoplastic cells of RCC may be arranged in clusters or nests, but in contrast to NVUC, the RCC nests are closely packed, imparting a solid pattern of growth, and are separated by a sinusoidal vascular network [5].

The NVUC will react with antibodies to cytokeratin 5 and 6, high-molecular-weight cytokeratin, and p63, allowing distinction from RCC, which is negative for these markers [5].

\section{Conclusions}

Nested variant of urothelial carcinoma is a histologically unique tumor, which should not be confused with florid von Brunn nest. In our case, invasion of the renal pelvis wall excludes the possibility of benign lesions of von Brunn type. Similarly, the presence of neoplastic infiltration excludes the type of nephrogenic adenoma. Our case concerns a woman, so it was not necessary to differentiate from a possible prostate cancer. In men immunomorphologic staining should be done with anti-tissue PSA. Diagnosis of NVUC in our case was made on the basis of morphologic features. The patient was cured of the neoplasm after surgery. To our knowledge this is the fourth case of NVUC with involvement of the renal pelvis.
Authors declare no conflict of interests.

\section{References}

1. Murphy WM. Diseases of the urinary bladder, uretes and renal pelves. In: Urological pathology 1997. Eble JN, Epstein J, Sesterhenn I, Sauter G. (eds.). WHO Pathology \& Genetics. Tumours of urinary system and male genital organs. 2004.

2. Stern JB. Unusual benign bladder tumor of Brunn's nest origin. Urology 1979; 14: 288-289.

3. Talbert ML, Young RH. Carcinomas of the urinary bladder with deceptively benign- appearing foci. A report of three cases. Am J Surg Pathol 1989; 13: 374-381.

4. Pusztaszeri M, Hauser J, Iselin C, et al. Urothelial carcinoma „,nested variant” of renal pelvis and ureter. Urology 2007; 69: 778: e15-e778.

5. Lau SK. Nested variant of urothelial carcinoma of the renal pelvis. Pathol Res Pract. 2009; 205: 508-512.

6. Tripodi S, Rocca BJ, Ambrosio MR, et al. Pelvic urothelial carcinoma with nested pattern of growth and an uncommon clinical presentation: a case report. Anal Quant Cytol Histol 2011; 33: 340-344.

\section{Address for correspondence}

Bolesław Kuzaka MD, PhD

Department of Urology,

Professor Witold Orlowski

Clinical Hospital,

Center for Medical Postgraduate

Education, Warsaw, Poland

tel. +48225021726

fax +48222148

e-mail: bolkuz@interia.pl 